\title{
The Effects of Meditation and Visualization on the Direct Mental Influence of Random Event Generators
}

\author{
Tayzia Collesso
}

Department of Psychology*, King's University College, Western University, Canada

MARIA ForRester

Faculty of Science*, Western University, Canada

\section{IMANTS BARUŠS}

Department of Psychology, King's University College, Western University, Canada baruss@uwo.ca

Submitted July 3, 2020; Accepted January 5, 2021; Published June 15, 2021

https://10.31275/20211891

Creative Commons License CC-BY-NC

*Tayzia Collesso is now at Faculty of Law, Western University; Maria Forrester is now at Mariposa Wellness Canada.

Abstract-Meditation and visualization exercises have been found to alter an individual's mood and perception, and it is hypothesized that these techniques will enhance one's ability to anomalously influence the function of a random event generator (REG) with the mind. This study comprises a control experiment and a second experiment with the administration of meditation and visualization exercises. There was no support for a significant deviation of the REG in the direction of the participants' volition in Experiment $1(t(29)=-1.26, p=.22$, two-tailed), but results revealed a significant deviation in the intended direction in Experiment $2(t(29)=2.66, p=.01$, two-tailed). Moreover, comparisons between cumulative deviations across both samples were found to be statistically significant, suggesting that the use of meditation and visualization exercises might promote significant deviations $\left(\mathrm{t}\left(5^{8}\right)=-2.69, p=\right.$ .009 , two-tailed). These analyses suggest that the use of meditation and visualization techniques in experiments that study direct mental influence may be beneficial for finding anomalous effects.

Keywords: meditation, visualization, random event generator, direct mental influence 
Discovery commences with the awareness of anomaly ... with the recognition that nature has somehow violated the paradigm-induced expectations that govern normal science. It then continues with a more or less extended exploration of the area of anomaly. And it closes only when the paradigm theory has been adjusted so that the anomalous has become the expected. (Kuhn, 1970, pp. 52-53)

\section{INTRODUCTION}

Psychokinesis (PK) is the apparent direct manipulation of animate or inanimate objects with the mind. It is a psi phenomenon, or a function of the mind that is anomalous in nature, as it cannot be explained by conventional science. There is a considerable amount of evidence that supports the occurrence of direct mental influence. However, the extent to which a person believes that physical manifestation can be affected directly by the mind depends on her boggle threshold, or what she is willing to accept as real (Barušs \& Mossbridge, 2017). It is important to note that our understanding of consciousness remains limited, and this arguably will persist if the scientific community continues to disregard the substantial evidence for anomalous phenomena and fails to give it sufficient consideration.

The Princeton Engineering Anomalies Research (PEAR) laboratory was developed to experimentally monitor direct mental influence within a controlled setting with an array of electronic devices. Effects on electronic devices are considered to be micro-PK. However, experiments also were conducted involving people-sized events. For example, a 6by 10 -foot random mechanical cascade was built to produce random events as 9,000 polystyrene balls were released into a transparent box filled with 330 perpendicular pegs; the polystyrene balls collected in the 19 slots at the base of the box so as to form a normal distribution. To test for the occurrence of direct mental influence, subjects were asked to attempt to mentally deviate the normal distribution to the left or to the right. A right-going intention run, a left-going intention run, and a baseline run were combined to form a session, and a series was composed of 10 or 20 sessions. The difference between left-going intention and right-going intention was statistically significant over 87 series and there was a probability of less than 5 in 100,000 that results occurred by chance (Dunne et al., 1988; Dunne \& Jahn, 1992; Barušs \& Mossbridge, 2017). 


\section{The Random Event Generator}

The use of electronic devices can be traced back to the 1970s, when Helmut Schmidt devised an electronic random event generator (REG) based on radioactive decay as a mechanism by which to study PK (Schmidt, 1971; Braud, 2003). There has been considerable REG research since that time. A comprehensive review can be found in Chapter 6 of Transcendent Mind by Imants Barušs and Julia Mossbridge (2017; see also Walach et al., 2020; Mertz, 2020). The first experiment reported in this paper mimicked the protocol developed in the PEAR lab. The REG consisted of an electronic device that produced a binary signal based on the reverse current across a diode. The output is set up in such a way that it can be thought of as a sequence of pluses and minuses with an equal chance of observing either a plus or a minus. The expected mean is 100 when the REG is set to 200 pulses per trial. A number of trials form a run, and a number of runs form a series. To test for the occurrence of direct mental influence, participants are asked to attempt to mentally deviate the randomly generated cumulative deviation line displayed on a computer screen away from the expected mean for a number of runs. The participant is asked to select a high or low intention for each independent run, and successful deviations occur when a participant's intention matches the output of the randomly generated line. Subsequently, the difference between the average scores of high-intention runs and low-intention runs, within a series, can be used to test for a statistically significant effect. Meta-analysis of PEAR laboratory experiments using the electronic REG over a period of 12 years, with 91 individual operators, found significant deviations for high-low differences in the intended direction $\left(z=3.81, p=6.99 \times 10^{-5}\right.$; Jahn et al., 1997). It is important to note that such anomalous effects are not necessarily caused by direct mental intention, even though we loosely talk about these experiments in that manner, but, in cases where participants have sufficient freedom to select or initiate runs, it could also be due to participants' precognitive abilities to anticipate the output from the REG. 


\section{Psychological Parameters Affecting Direct Mental Influence}

Jim Carpenter has theorized that the capability to affect physical manifestation with the mind is a non-conscious ability, lying at the boundary of one's ordinary mental capacities and explicit awareness and intention. The premise is that psi phenomena lie at the primary stage of experience and that all experience, intention, and action emerge from psi processes. Our non-conscious intentions are deemed to be primary as these intentions function to help our psyche select and understand the nature of our experiences, and to initiate a response to those experiences (Carpenter, 2004). Correspondingly, in emotional presentiment studies, certain networks of meaning and affect have been shown to become aroused prior to sensory stimulation, indicating that parts of our psyche appear to perceive a stimulus before it is present, hence, regarding such perception as "first sight" (Carpenter, 2004). It should be noted that the presentiment studies look at ESP and not PK specifically; it may be plausible, however, that PK functions in a similar manner to ESP (Carpenter, 2004). As psi processes are conceived to function in a parallel manner to conscious processes, Jim Carpenter (2004) has posited that direct mental influence in the intended direction may be possible for individuals who can synchronize non-conscious intentions with conscious intentions.

The mind apparently is able to appraise extrasensory information, but tuning into relevant material, as well as maintaining intention stability and turning away from irrelevant material, are important for producing significant REG deviations. Conflicted intentions, on the other hand, are believed to produce smaller deviations from chance (Carpenter, 2004). If individuals are able to tune in to an experience and accept the flow of events, it is believed that direct mental influence in the intended direction will be facilitated. Contrarily, if an individual is resistant to the flow of events, perhaps due to an underlying factor, such as frustration or fear, effects in the unintended direction appear to be produced (Carpenter, 2004). In an ESP study conducted by Carpenter (1971), subjects were asked to perform an extrasensory perception guessing task. Half of the targets were placed with emotionally evoking material while the other half were placed with blank cards. The subjects who were low in anxiety were able to accurately guess the targets that 
were placed with arousing material, indicating that these subjects may have had an implicit interest in the emotionally evoking material and, perhaps, were able to tune into its relevancy. The subjects who were high in anxiety, however, psi-missed on the emotionally evoking material and did not choose the targets with arousing material, almost as if these subjects' non-conscious intentions were to avoid a potentially dangerous event (Carpenter, 1971). If PK processes are analogous to ESP, this suggests that a certain level of openness and unattachment may be necessary to tune into relevant material. In other words, if an individual places too much emphasis on correctly completing the task or performs an excessive amount of cognitive analysis, an effect in the unintended direction may be observed.

While negative emotions tend to correspond with deviations of the REG in the unintended direction (Carpenter, 2004), there is research that supports that emotions, such as enthusiasm and feelings of connectedness with the machine, have been related to successful deviations. The case of Susan Padfield is an example of research concerning emotions of connectedness (Barušs \& Mossbridge, 2017). Susan Padfield was able to influence the movement of a light mobile in laboratory settings for multiple observers, and she indicated that she felt as though she had merged with the mobile on a mental level and was able to create its future state (Padfield, 1980). Perhaps, the ability to maintain intentional stability is facilitated by positive emotions. Similarly, Jahn et al. (1997) found that there was a commonality to be found in PEAR lab experiments: The most successful operators tended to describe the devices in anthropomorphic terms, describing the machine as if having a bond with it or empathizing with it. Certain individuals may also be more gifted or motivated to engage psi abilities as a result of doing creative work, and this may be why individuals who were high in creativity were also more likely to produce significant evidence for psi in Ganzfeld telepathy experiments (Barušs \& Mossbridge, 2017). Furthermore, the fact that creativity may have an impact on psi processes coincides with the belief that maintaining a certain level of openness may promote direct mental influence in the intended direction. 


\section{Direct Mental Influence Without Intent}

In the same way that the emotions and cognitions of subjects have an effect in direct mental influence experiments, it is also important to consider the emotions of others, and a possible experimenter effect. In fact, some studies have shown that the REG appeared to deviate in accordance with the emotional states of participants who were focusing on alternative tasks (Broderick \& Goertzel, 2014). The FieldREG is a way of conceptualizing the output from the random event generator as a single bit-stream to test for PK without directed conscious intention. One way of thinking about such studies is to imagine that the FieldREG can be influenced by a consciousness field that interacting participants may generate (Nelson et al., 1996). Consistent with such ideas, Nelson et al. (1998) discovered that there were significant FieldREG deviations that correlated with the emotional engagement and enthusiasm generated by participants while visiting several venues, including ancient Egyptian sites and the New York City Opera. Intellectual engagement, on the other hand, did not produce any significant effects. As a second example, Jahn et al. (2006) studied the effects of a Japanese healing technique on subjects in human-machine experiments and discovered that the outputs of FieldREG data tended to display mean shifts that deviated from what is expected by chance when there was "a high degree of emotional resonance among the participants" (p. 2). As a third example, Roger Nelson ran a FieldREG during a field trip to watch a sunset across a lake. As the sun set, the participants became silent and directed their attention to the setting sun. If the one-hour segment bracketing the time of the sunset is regarded as a pre-defined dataset, then the striking positive deviation of the FieldREG that was noted during that timeframe had a probability of occurrence of $p=.008$ (Barušs, 2007).

Even though the direction of the deviation cannot be predicted with the FieldREG, it appears that high levels of positive emotions tend to correspond to directional deviations in REG functioning (but see also Varvoglis \& McCarthy, 1986). This leads to a question that we hoped to answer: What exactly are the relevant parameters of such positive emotions? In addition, we would like to know whether a participant's nonconscious and incidental influence on FieldREG functioning is 
correlated with the results of deliberate influence on REG functioning. In this study we aim to investigate whether individuals who can produce FieldREG deviations are also more likely to produce REG deviations for intentional runs.

\section{Meditation as an Aid}

Any discussion of meditation needs to begin with the acknowledgment of just how vast the meditation literature is and how large the range of psychological and physiological states that accompany meditation is (Barušs, 2020). In terms of regulating emotion, Margolin et al. (2011) have noted that regular identification with anxiety creates a barrier to experiencing peace and joy, and some forms of meditation may be used to reduce this anxiety. Meditation may also be used to induce both physical and mental stillness in an altered state of consciousness. Likewise, it has been found to enhance one's self-awareness (Margolin et al., 2011). Silencing excessive mental noise or rational thoughts, as well as heightening one's awareness of a deeper self, may increase an individual's capacity to tune in to relevant information. If meditation is able to reduce the extent to which negative emotions affect our awareness and allow us to align our unconscious intentions with our conscious intentions, then this could be a key for the study of direct mental influence.

Recent literature in neuroscience suggests that meditation practices appear to alter brain chemistry, structure, and function in a more permanent manner as well. Specifically, it was reported that long-term meditation practices have an effect on brain regions that are associated with emotion and attention. For example, meditation has been linked with increased selective attention capacity, which is an enhanced ability to focus on a certain outcome while silencing undesired outcomes. Moreover, mindfulness meditation has been correlated with diminished activity in the amygdala, which is associated with the production of fear and anxiety (Margolin et al., 2011). Both of these effects would appear to benefit direct mental influence in the intended direction.

Some types of meditation and visualization practices draw on the notion that the mind has an untapped potential to create, and this 
potential can be brought into awareness so that desired states become reality. Specifically, conscious visualization is believed to have the ability to create thoughts, emotions, and events in one's phenomenal world in accordance with one's ambitions and goals (Margolin et al., 2011). In some forms of meditation, the distinction between subject and object, and the observer and the observed, becomes blurred. Loving-kindness and compassion meditations also are of particular interest here as they generate a consistent emotional tone that is exclusive of other types of affect (Fox et al., 2016). The consistent emotional tone is perhaps generated as participants are asked to project positive emotions toward others or in reference to no subject at all.

If meditation and visualization are able to transform the brain, one's mood, and one's perception, then it is possible that applying these techniques to PK experiments may facilitate direct mental influence in the intended direction. In a series of experiments in which participants were asked to direct their attention toward or away from a two-slit optical device, it was found that those who identified themselves as meditators, without further specification of the type of meditation that they practiced, were better able to influence the device when requested to direct their attention toward the device than those who did not identify themselves as meditators (Radin et al., 2012). One of the strategies in Experiment 2 in this study was to prime feelings of love by leading participants through a guided imagery meditation as one of a number of manipulations that could increase the likelihood of finding a positive REG deviation.

\section{Hypotheses}

The purpose of these studies is to contribute to the expanding literature on consciousness by exploring the psychological correlates of direct mental influence and the role that meditation and visualization may have in enhancing anomalous manipulation. Experiment 1 will act as a control sample for Experiment 2, which introduces the meditation and visualization component to the study. The comparison of both samples will make it easier to identify any relevant parameters. 


\section{Experiment 1}

1. It is hypothesized that there will be a significant deviation in the intended direction.

2. It is hypothesized that trials without directed conscious intention, measured by the REG running in FieldREG mode, will predict subsequent trials completed with intention as measured by the REG in PEAR Classic mode.

3. The Beliefs About Consciousness and Reality Questionnaire (BACARQ) is used for measuring transcendental beliefs. It is hypothesized that individuals with a high composite score on the BACARQ will be more likely to deviate the REG in the intended direction.

4. The Expressions of Spirituality Inventory (ESI) is used for measuring spirituality. It is hypothesized that individuals with a high composite score on the ESI will be more likely to deviate the REG in the intended direction.

5. It is hypothesized that individuals who are in an altered state of consciousness, as indicated by the Phenomenology of Consciousness Inventory $(\mathrm{PCl})$, will be more likely to deviate the REG in the intended direction.

6. It is hypothesized that those who identify with the machine or feel a transcendent unity with it will be more likely to deviate the REG in the intended direction.

7. It is hypothesized that those who think they can alter the machine also will be more likely to deviate the REG in the intended direction.

\section{Experiment 2}

1. The meditation and visualization techniques used will aid in promoting successful deviations in that there will be significantly more deviations in the intended direction in comparison with Experiment 1.

2. Individuals who experience feelings of love, as measured by the Phenomenology of Consciousness Inventory, will be more likely to deviate the REG in the intended direction.

3. Individuals scoring high in rationality, as measured by the Phenomenology of Consciousness Inventory, will be less likely to deviate the REG in the intended direction. 


\section{GENERAL METHOD}

\section{Participants}

Participants were volunteers recruited from Western University, King's University College consciousness-related courses, the Psychology 1,000 participant pool, and the community. There was a total of 60 participants for both experiments, with 30 participants in each sample. In Experiment 1, the mean age was 22.9 years $(S D=6.9$, age range: 18-55). Eighteen participants were recruited from the participant pool whereas 12 participants were volunteers outside of the participant pool. The mean age for Experiment 2 was 29.1 years $(S D=10.5$, age range: 19-69), and the sample consisted primarily of individuals from the community $(n=18)$. Students currently enrolled in consciousnessrelated courses and individuals who expressed an interest in the study of consciousness were targeted due to the fact that expectation and beliefs about consciousness are presumed to be important in terms of being receptive to anomalous phenomena and of deviating the REG in the intended direction. Although the participants did not receive monetary compensation, participants from the introductory psychology pool were granted course credit for taking part in the study.

\section{Materials}

Desktop Computer. A custom desktop computer, built by Superior Computers and linked to a Sony computer monitor, an ergonomic keyboard, and an ergonomic mouse, was used for running specialized REG software with which study participants interacted.

Random Event Generator (REG). The REG used in this study was an electronic device manufactured by Psyleron that produces random binary events by measuring the reverse current across a diode that is produced by quantum mechanical tunneling. This quantum process produces random sequences of one of two possible outcomes which can be displayed on a computer to which it is attached, in order to determine if the sequence conforms to random alternation. Both FieldREG software and PEAR Classic REG software are loaded on the computer and are used to register input from the REG, as well as display the data on the computer screen in the manner of a cumulative deviation graph (Psyleron, 2009). The FieldREG software is used to 
collect data without any intention to move the randomly generated cumulative deviation line up and down, whereas the PEAR Classic REG software is used to collect data with such intention. In the present study we investigated any correlations between cumulative averages for the FieldREG and PEAR Classic REG. Since only relative measures and correlations were used in this study, no control or calibration runs were necessary, nor were any carried out. It should be noted that if direct mental influence does occur, as previous research has indicated, then even control and calibration runs are being influenced by the experimenter and anyone else who becomes aware of such runs.

PseudoREG. The PseudoREG mimics the output of an REG by using the random number generator on a computer rather than on the REG. The PseudoREG, instead of the REG, was inadvertently used with the PEAR Classic REG software in Experiment 1.

Relaxation Meditation Script. A 2- to 3-minute relaxation meditation was used in Experiment 2 to redirect thoughts about external events toward internal occurrences within the body. The primary goal of this relaxation meditation was to promote tension identification as well as tension reduction. It is assumed that the release of tension would encourage the release of other negative emotions, such as frustration, fear, and anxiety, and would also increase awareness. The script can be found in Appendix A.

Visualization Exercise Script. A 3- to 5-minute guided imagery exercise was used following the relaxation meditation in Experiment 2 and the script can be found in Appendix B. The visualization exercise was employed to stimulate imagination and further induce relaxation as well as positive emotions, including promoting feelings of love.

Visualization Suggestions. Prior to interacting with the REG in Experiment 2, participants were introduced to the basic components of the PEAR Classic REG software and were given strategies that were believed to promote deviations in the intended direction. These suggestions can be found in Appendix C. Participants were encouraged to apply visualization techniques and to attach positive qualities to the randomly generated line. Participants were also advised to avoid harboring feelings of frustration when the line deviates in a manner inconsistent with the individual's intended direction.

Environmental Manipulations. In an attempt to produce a more 
relaxing, inspiring, and all-around positive environment in Experiment 2, a potted plant was placed on the desk and posters that illustrated woodland imagery, along with other visuals intended to encourage calmness and imaginative thinking, were strategically mounted on the walls. Moreover, the overhead lights of the lab room were switched off for the duration of the experiment, and instead, two desk lamps were used to illuminate the desired areas of the room.

\section{Measures}

Demographics and Attitudes Form. This form was given at the beginning of both experiments and contains questions about the participant's age, gender, education, religious views, and frequency of religious practice. It also contains a five-point Likert-type question pertaining to the participant's belief in their ability to alter the functioning of the REG with one being "definitely not" and five being "definitely yes." This form can be found in Appendix D.

Beliefs About Consciousness and Reality Questionnaire (BACARQ). Imants Barušs and Robert Moore developed the BACARQ in 1987 as a self-report questionnaire with 38 items (Barušs \& Moore, 1992, 1998). Questions one to eight use a four-point Likert scale with one representing "Definite No" and four representing "Definite Yes." Questions 9 to 38 use a seven-point Likert scale with one representing "Strongly Disagree" and seven representing "Strongly Agree." The seven dimensions that are measured with this questionnaire include Antiphysicalism, Religiosity, Meaning, Extraordinary Experiences, Extraordinary Beliefs, Inner Growth, and Transcendentalism, which is the global scale made up of all items. A higher score on the global scale represents more transcendental beliefs. The Cronbach's alpha for all of the items totaled to form a single scale was $\alpha=0.95$ with items 9 , 19, 24, 29, and 32 being reverse-scored items. A sample item from the Antiphysicalism dimension is Item 9, "There is no reality other than the physical universe." The questionnaire was used in both experiments.

Expressions of Spirituality Inventory (ESI). Douglas MacDonald developed the revised version of the ESI in 2000 (2000a, 2000b); and the inventory contains a total of 32 items. This instrument uses a five-point Likert-type scale from zero to four, with zero being "Strongly Disagree" 
and four being "Strongly Agree." The inventory measures five descriptive dimensions of spirituality including an Experiential/Phenomenological dimension, Cognitive Orientation toward Spirituality, Existential WellBeing, Paranormal Beliefs, and Religiousness. Higher scores represent more spiritual beliefs. Cronbach's alpha for the individual dimensions varied between $\alpha=0.80$ and $\alpha=0.89$. Reverse-scored items are items $3,8,13,18,19,23$, and 28. A sample item from the Experiential/ Phenomenological dimension is, "I have had an experience in which I seemed to be deeply connected to everything." The inventory was used in both experiments.

Phenomenology of Consciousness Inventory (PCI). Ronald Pekala developed the $\mathrm{PCl}$ in 1991 (1991a, 1991b). This inventory is a retrospective report including 53 items that are used to assess the state of consciousness a participant found themself in during a specific time period using a seven-point scale with opposing statements on opposite ends of each scale. The $\mathrm{PCl}$ was used in both experiments to evaluate a participant's state of consciousness while the FieldREG software and the PEAR Classic REG software registered input from the REG or PseudoREG. The scale includes 21 subscales and dimensions of subjective experience: Joy, Sexual Excitement, Love, Anger, Sadness, Fear, Body Image, Time Sense, Perception, Meaning, Imagery Amount, Imagery Vividness, Direction of Attention, Absorption, Self-Awareness, Altered State, Internal Dialogue, Rationality, Volitional Control, Memory, and Arousal. All of the subscales were checked to see if they could predict the difference between high- and low-intention runs, but the altered states subscale was of particular interest. The Cronbach's alpha for the individual subscales ranged from $\alpha=0.72$ to $\alpha=0.91$, and a sample item from the altered state subscale is: "My state of consciousness was not any different from or more unusual than what it ordinarily is o 123456 . I felt in an extremely different and unusual state of consciousness."

Questions about Experience. This form was given at the end of both experiments and includes an open-ended question about the participant's experience while interacting with the REG, followed by two Likert-type questions with a five-point scale, where one represents "definitely not" and five represents "definitely yes." The first Likert-type question measures whether or not participants felt that they had been 
able to alter REG functioning, and the second measures a participant's sense of connectedness with the REG. These three questions can be found in Appendix E.

Psyleron FieldREG Software. FieldREG software that was developed by Psyleron and loaded on the laboratory computer, was used to gather and quantify non-intentional data from the REG while participants were filling out the questionnaire and inventories at the beginning of the session. The REG was accumulating data passively.

Psyleron PEAR Classic REG Software. PEAR Classic REG software that was developed by Psyleron and loaded on the laboratory computer, was used to gather and quantify data from the REG or PseudoREG during the intentional runs and, thus, to measure possible direct mental influence. The PEAR Classic program was used for both experiments and it is a computer program that is a recreation of the original protocols used at the Princeton Engineering Anomalies Research laboratory at Princeton University. Each participant was asked to perform a total of 20 runs, and a balanced series was required so that participants were asked to perform 10 high-intention runs and 10 lowintention runs. A participant's performance is shown on the computer screen in real-time through the use of a cumulative deviation graph. The difference between cumulative averages for high-intention runs and low-intention runs was used as a dependent measure.

\section{Procedure}

Experiment 1 and Experiment 2 were conducted in a designated Psychology Laboratory on the King's University College campus after receiving appropriate ethics approval for research with human subjects. Initially, each participant was greeted and asked to review and sign a consent form. The consent form contained information about the purpose of the study and the role of the REG and FieldREG in such experiments. The participant was informed that FieldREG software would be registering input from the REG as it ran passively while three forms were completed in the following order: a demographics and attitudes form, the BACARQ, and the ESI. Subsequently, the REG was turned off and the participant was asked to fill out the $\mathrm{PCl}$ based on her subjective experience while filling out the initial survey package. 
The FieldREG software was turned off while the participant filled out the $\mathrm{PCl}$ because the $\mathrm{PCl}$ was administered to measure the participant's subjective experience while the FieldREG software was running passively.

Experiment 2 differs from Experiment 1, in that a meditation component was added to the procedure in Experiment 2 prior to introducing a participant to the PEAR Classic program. The PEAR Classic program is the program that was used when a participant was asked to consciously deviate a randomly generated line that was displayed on the computer screen toward their pre-stated intention, based on their own volition, for a total of 20 runs. A balanced series was required, and therefore it was necessary that the participant complete 10 high-intention runs and 10 low-intention runs in an order of their choice. "High intention" and "low intention" are operationally defined as the behavioral selection of high-intention and low-intention runs, respectively. During a high-intention run, the participant's goal was to increase the value of the mean, and in a low-intention run, the participants' goal was to decrease the value of the mean. If the difference between the high and low mean cumulative deviations is statistically different from the expected mean, then it would appear that there is a correspondence between intention and the functioning of the electronic device through either direct mental influence or precognition.

Once the series of runs was completed in both Experiment 1 and Experiment 2, the PEAR Classic program was turned off, and the participant was asked to fill out the $\mathrm{PCl}$ for a second time, in order to evaluate the nature of a participant's subjective experience while interacting with the PEAR Classic program. After that, the participant was asked to fill out a final form, which consisted of three questions about their experience with the REG which were administered to discover any psychological variables that may be associated with significant deviations. Finally, the participant was asked to review a debriefing form, and any participants from the participant pool were given an opportunity to complete the bonus assignment in order to receive course credit. 


\section{Design}

Correlations between the predictor variables and the criterion variable were analyzed in order to identify any significant relationships in both experiments. FieldREG mean scores, BACARQ composite scores, ESI cumulative scores, $\mathrm{PCl}$ scale scores, scores representing connectedness with the REG, and scores representing belief about REG influence served as predictor variables, and the mean difference between high- and low-intention runs served as the criterion variable; all variables are continuous. Moreover, the data from the sample in Experiment 1 and the sample in Experiment 2 were compared using an independent samples $t$-test in order to determine if the sample means differed significantly, potentially indicating that the changes in participants, equipment, and protocol in Experiment 2 may promote successful deviations in the intended direction.

\section{EXPERIMENT 1}

\section{Method}

In Experiment 1, the REG was used as the signal source for the Psyleron FieldREG software and the PseudoREG was used as the signal source for the PEAR Classic REG program. The REG was set up at 5 trials per second with each trial consisting of 200 bits for the Psyleron FieldREG software. For the PEAR Classic program, the PseudoREG was set up at 4 trials per second, 50 trials per run, and 1,000 total trials. There were no environmental manipulations, no meditation and visualization exercises, nor visualization suggestions prior to participants interacting with the PEAR Classic program.

\section{Results}

When testing our hypotheses, adjustments were not made for multiple analyses since the hypotheses were prestated, and, furthermore, none of them was confirmed for a critical value of .05. Where we do find effects in post hoc analyses, the results need to be interpreted with care, given that the probability of a Type I error increases with the number of analyses that are carried out. However, adjusting for multiple analyses, for instance with a Bonferroni correction, increases 
the probability of Type II errors. So, unless otherwise noted, we simply report what we found for the reader to consider.

Hypothesis 1. In order to determine if there was a significant deviation in the intended direction, the difference between cumulative scores for high intention runs and cumulative scores for low intention runs was analysed using a one-sample Student's $t$-test. The mean difference for high- and low-intention runs was -.083 $(S D=.36)$ and the $t$ statistic was not significant, with $t(29)=-1.26(p=.22$, two-tailed). These findings do not provide support for direct mental influence in the intended direction.

Hypothesis 2. A stepwise linear regression was used to determine if the criterion variable, the mean difference between high- and lowintention runs, could be predicted from the values of the predictor variables. Hypothesis 2 was tested by examining the correlation between the mean difference between high- and low-intention runs and FieldREG mean scores. FieldREG mean scores are representative of trials without directed conscious intention, while the mean differences between high- and low-intention runs are representative of trials with intention. The correlation between the predictor variable and the criterion variable was $r(28)=.06, p=.75$ (two-tailed), but the predictor variable was not entered into the regression equation because the probability of the correlation between FieldREG averages and the mean differences between high- and low-intention runs was above .05. In other words, trials without directed conscious intention did not predict trials completed with intention.

Hypothesis 3. The correlation of composite scores on the Beliefs About Consciousness and Reality Questionnaire with the criterion variable was insufficiently significant for the variable to be entered into the regression equation, $r(27)=-.10, p=.61$ (two-tailed). Therefore, individuals with a high composite score on the BACARQ, which is indicative of transcendental beliefs, were not more likely to deviate the REG in the intended direction.

Hypothesis 4. The largest correlation of a scale from the Expressions of Spirituality with the criterion measure was that of Cognitive Orientation toward Spirituality with $r(28)=-.12, p=.54$ (two-tailed). These findings provide no evidence in support of high scores on the ESI being indicative of an ability to deviate the REG in the intended direction. 
Hypothesis 5. In order to determine if an altered state of consciousness would be indicative of an ability to deviate the REG in the intended direction, the altered state subscale scores on the second administration of the $\mathrm{PCl}$ were used as a predictor variable. The predictor variable was not entered into the regression equation because the correlation between the predictor variable and the mean difference between high- and low-intention runs, $r(27)=-.04, p=.85$ (two-tailed), was above .05. It is important to note that a new binary variable was created representing the direction of the deviation. A 'one' signifies a positive difference between high- and low-intention runs, or a deviation in the intended direction, whereas a 'zero' signifies a negative difference, and therefore a deviation in the direction opposite to intention. The correlation between the altered states subscale scores on the second administration of the $\mathrm{PCl}$ and a positive difference between high and low intention runs was statistically significant, $r(27)$ $=.40, p=.03$ (two-tailed). When checking for a difference between the two groups using ANOVA, Levene's test for equality of variances was significant, and therefore, when equality of variances was not assumed, the independent samples $t$-test was not significant, $t(10.92)=-1.89, p$ $=.09$ (two-tailed). Thus, while suggestive, there is insufficient support that an altered state of consciousness while interacting with the REG is correlated with a deviation in the intended direction.

Hypothesis 6. The correlation between the criterion variable and the scores representing connectedness with the REG was not significant, $r(28)=.11, p=.56$ (two-tailed), and therefore the predictor variable was not entered into the regression equation. In other words, participants who identify with or feel a transcendental unity to the REG, are not more likely to deviate the REG in the intended direction. In a follow-up analysis, however, it was found that the scores representing connectedness with the REG were correlated with the love subscale on the second administration of the $\mathrm{PCl}, r(28)=.65, p$ $<.001$ (two-tailed). Moreover, the correlation of the criterion variable with a new variable, the change in the love subscale from the first administration of the $\mathrm{PCl}$ to the second administration, gives $r(27)$ $=.39(p=.04$, two-tailed) (Figure 1$)$. That means that the change in the love subscale accounted for $15 \%$ of the variance in the mean 


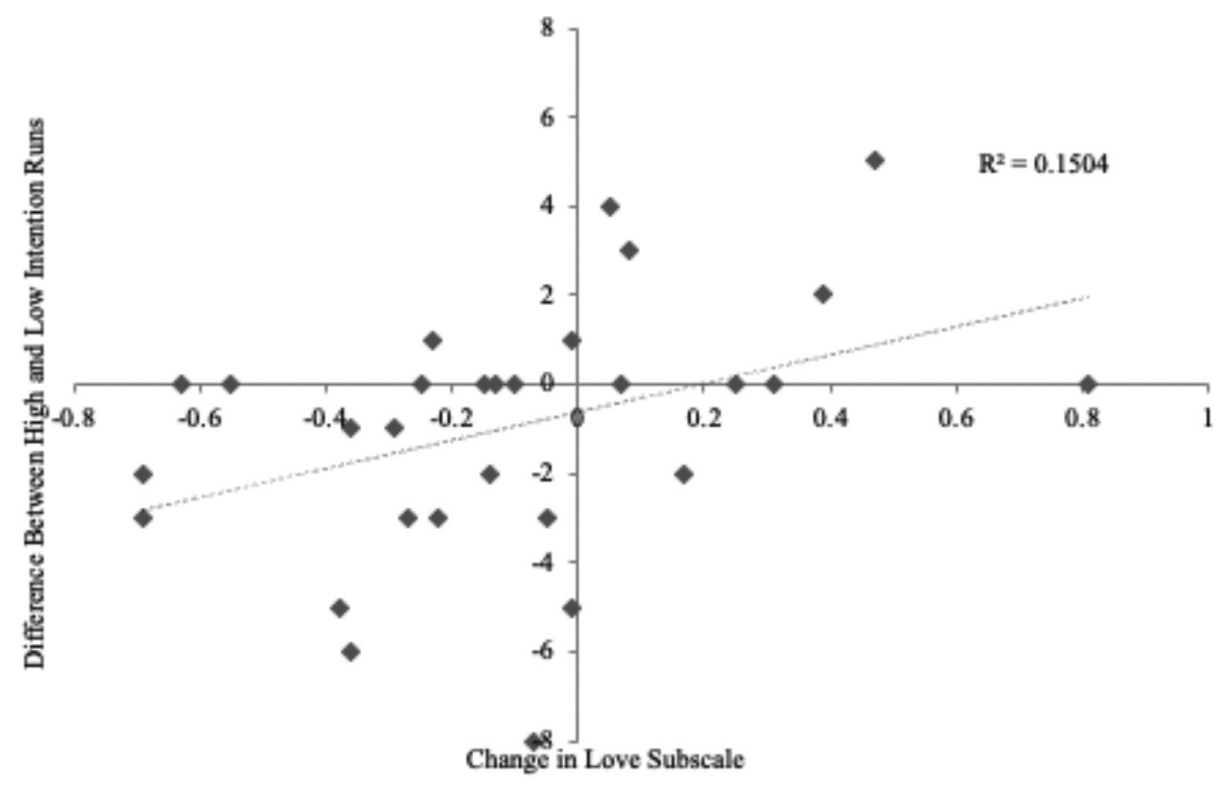

Figure 1. The relation between the change in the love subscale and the difference between high- and low-intention runs.

difference between high and low intention runs (Figure I). If love is associated with a feeling of connectedness, and a change in love or an increase in love scores is associated with the difference between high and low intention runs, then a significant correlation between scores representing connectedness with the REG and the criterion variable could be revealed if there were more statistical power. This relationship may also be mediated by love. It is important to note that any such relationships are not necessarily due to the presence of anomalous variables. Participants who 'succeeded' in creating a deviation in the intended direction could simply have subsequently reported having had more immersive and positive experiences.

Hypothesis 7. To determine if beliefs about REG influence would predict an ability to deviate the REG in the intended direction, the belief score, which was accumulated prior to any interaction with the REG, was analyzed for statistical significance. The predictor variable was not entered into the regression equation, however, as it was insufficiently 
TABLE 1

Correlations, Significance, and Sample Size of Predictor Variables with the Difference Between High- and Low-Intention Runs

\begin{tabular}{lccc}
\hline \hline Predictor Variable & $\begin{array}{l}\text { Pearson } \\
\text { Correlation }\end{array}$ & $\begin{array}{c}\text { Significance } \\
\text { (two-tailed) }\end{array}$ & N \\
\hline FieldREG Average & .06 & .75 & 30 \\
BACARQ Composite Score & -.10 & .61 & 29 \\
ESI Cognitive Orientation & -.12 & .54 & 30 \\
Altered State Subscale Score & -.04 & .85 & 29 \\
Resonance Score & .11 & .56 & 30 \\
Belief Score & -.19 & .31 & 30 \\
\hline
\end{tabular}

significant, $r(28)=-.19(p=.31$, two-tailed). A summary of the findings for each predictor variable can be found in Table 1.

Post Hoc Analyses. Significant correlations were found between FieldREG mean scores and education, as well as rationality $(r=-.401$, $p=.028, n=30$ ). Now we do need to explicitly take into account the fact that there were multiple analyses. We did this by splitting the sample into two equal-sized parts to determine if the relationship was maintained in both samples despite considerable loss of statistical power. One way to think of this is to conceptualize one half of the sample as the experiment in which the effect was found, and the other half of the sample as a replication experiment. Education lost its significance on each of the two random samples, but the correlation between rationality and FieldREG mean scores remained significant in one of the two random samples, $r(13)=-.81, p<.01$ (two-tailed), although the effect was not replicated in the other, $r(13)=.20, p=.47$ (two-tailed). Nonetheless, because the correlation between rationality and FieldREG mean scores showed up again in Experiment 2, it is reported here. 


\section{Discussion}

There was no evidence in support of a deviation in the intended direction. Furthermore, none of the individual characteristics hypothesized were indicative of an ability to deviate the REG in the intended direction. The correlation between FieldREG mean scores and rationality provides some evidence suggesting that excessive cognitive analysis may have a noticeable effect on an REG. The love subscale on the second administration of the $\mathrm{PCl}$ was correlated with scores representing connectedness with the REG, and a change in love between both administrations of the $\mathrm{PCl}$ was significantly correlated with the difference between high- and low-intention runs. In other words, the unification of an individual's sense of identity with the machine or empathizing with the machine may facilitate the information transfer between one's consciousness and the REG through the presence of love (Jahn, 1996).

In terms of limitations, the PseudoREG was used as the electronic noise source for trials with intention as a result of a programming error. Jahn et al. (1987) notes, however, that the PseudoREG and REG are identical in terms of the feedback provided and the experimental protocol used. Moreover, the results of 29 experimental series with a PseudoREG at the PEAR lab, confirmed the ability of operators to produce significant deviations using such experimental protocols (Jahn et al., 1987).

Feelings of love and silencing of excessive cognitive noise may improve the rate at which successful deviations occur, and the meditation and visualization component added in Experiment 2 will explore the effect of priming such emotions and perceptions.

\section{EXPERIMENT 2}

\section{Method}

The designated psychology laboratory at King's University College was strategically decorated in an attempt to produce a relaxing environment in Experiment 2. Moreover, an unsystematic effort was made to recruit participants, such as natural healers, who believed that they had some ability to directly manipulate physical reality. The 
Psyleron FieldREG software was set up at 5 trials per second with each trial consisting of 200 bits, and the FieldREG program registered input while participants filled out the initial survey package. After completion of the first $\mathrm{PCl}$ form, and prior to interacting with the PEAR Classic program, participants were guided through a 2- to 3-minute relaxation meditation, followed by a 3- to 5-minute guided imagery exercise. Subsequently, participants were offered visualization suggestions and strategies that were intended to promote direct mental influence in the intended direction. PEAR Classic REG trials then commenced. For those runs, the REG was set up at 4 trials per second, 50 trials per run, and 1,000 total trials.

\section{Results}

Hypothesis 1. Prior to confirming whether or not the meditation and visualization techniques used in Experiment 2 promoted successful deviations of the REG, a two-tailed single-sample $t$-test was conducted to test the mean difference between cumulative scores for high intention-runs and low-intention runs and the constant value zero, to determine if participants were significantly able to alter the functioning of the REG. A statistically significant difference was found, $t(29)=2.66(p=.01$, two-tailed, Cohen's $d=0.49)$, suggesting that there was a significant separation in the intended direction (Figure 2). In an attempt to establish whether or not participants who engaged in meditation and visualization exercises exhibited more successful deviations than participants who did not, comparisons were made with Experiment 1. An independent samples t-test was conducted comparing mean differences for high- and low-intention runs in both samples. A significant difference was observed between Experiment $1(M=-.08$, $S D=.36)$ and Experiment $2(M=.15, S D=.31)$, with $t(58)=-2.69(p=$ .009 , two-tailed). Since the separation of high and low intention runs is the single overall outcome measure, no correction for multiple analyses is required. This suggests that some of the differences between the conditions of Experiment 1 and Experiment 2, such as participating in meditation and visualization exercises prior to interacting with the REG, may enhance the likelihood of successful deviations.

Hypothesis 2. In Experiment 1, a change in love across both administrations of the $\mathrm{PCl}$ was correlated with the mean difference 


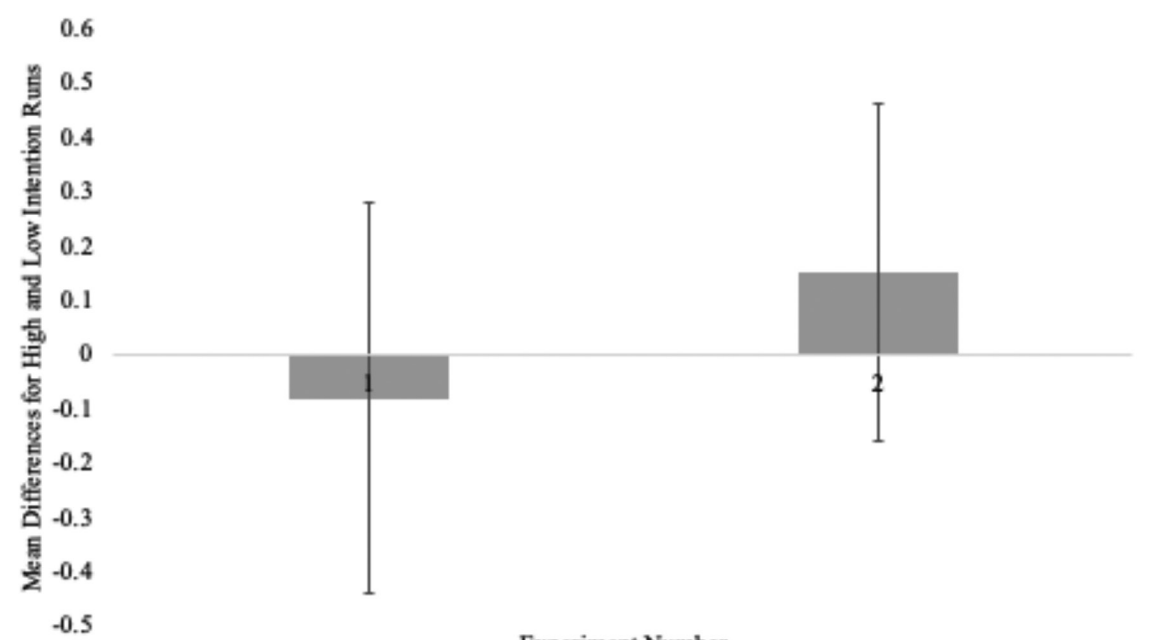

Experiment Number

Figure 2. Mean differences for high- and low-intention runs in Experiment 1 and Experiment 2. Error bars indicate the standard deviation.

for high and low intention runs. However, these findings were not replicated in Experiment 2, $r=-.12, p=.55$ (two-tailed). Moreover, the correlation between love subscale scores on the second administration of the $\mathrm{PCl}$ and the mean difference for high and low intention runs was not significant, $r(28)=-.26, p=.16$ (two-tailed) and in the opposite direction. A one-way analysis of variance was conducted to investigate the differences between scores for the love subscale on the second administration of the $\mathrm{PCl}$ for both experiments. The results revealed a statistically significant difference between the love subscale scores obtained from the second half of the two experiments, $F(1,58)=4 \cdot 31$, $p=.04$ (two-tailed) (Figure 3). This difference was in the expected direction with $M=4.57(S D=4.14)$ for Experiment 2 in comparison with $M=2.63(S D=2.98)$ for Experiment 1 . The difference between love scores on the first administration of the $\mathrm{PCl}$ was not different from Experiment 1 to Experiment 2, with $F(1,57)=.21(p=.65)$, thereby providing some confirmation that the manipulation had the intended effect of increasing feelings of love. Thus, given that participants in Experiment 2 were more likely to successfully deviate the REG, there is some support that feelings of love could be a factor in direct mental influence. 


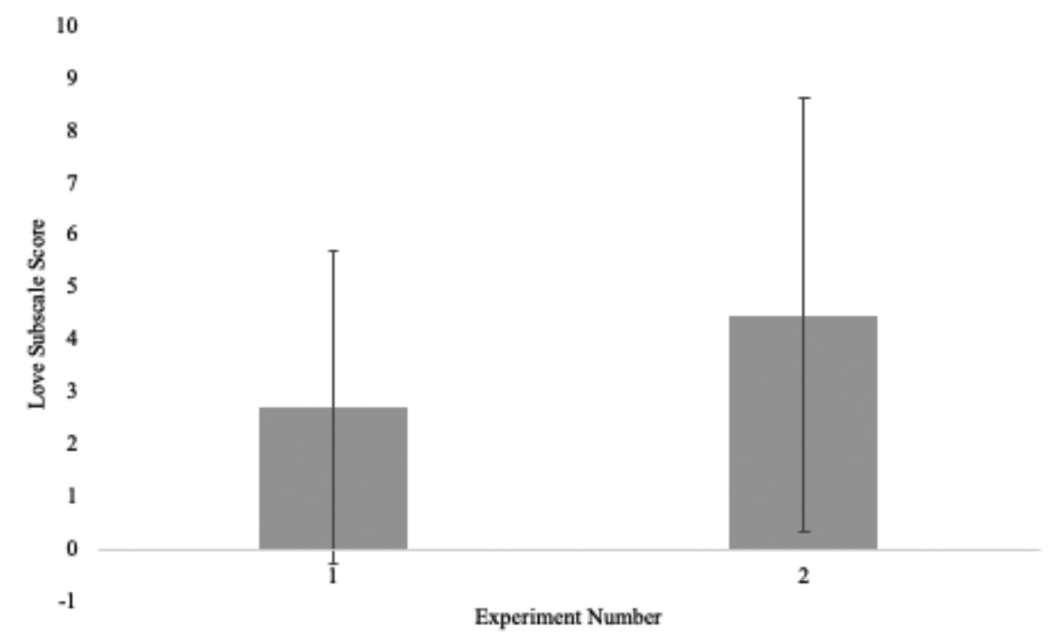

Figure 3. Love subscale scores for Experiment 1 and Experiment 2. Error bars indicate the standard deviation.

Hypothesis 3. Participants who scored high in rationality on the first administration of the $\mathrm{PCl}$ were not less likely to deviate the REG in the direction of their previously stated intent, $r(28)=-.28, p=.13$ (twotailed). There was, however, a significant correlation between rationality and FieldREG averages, $r(28)=-.38, p=.04$ (two-tailed) (Figure 4). This inverse effect was also significant in Experiment 1 , and it is suggestive

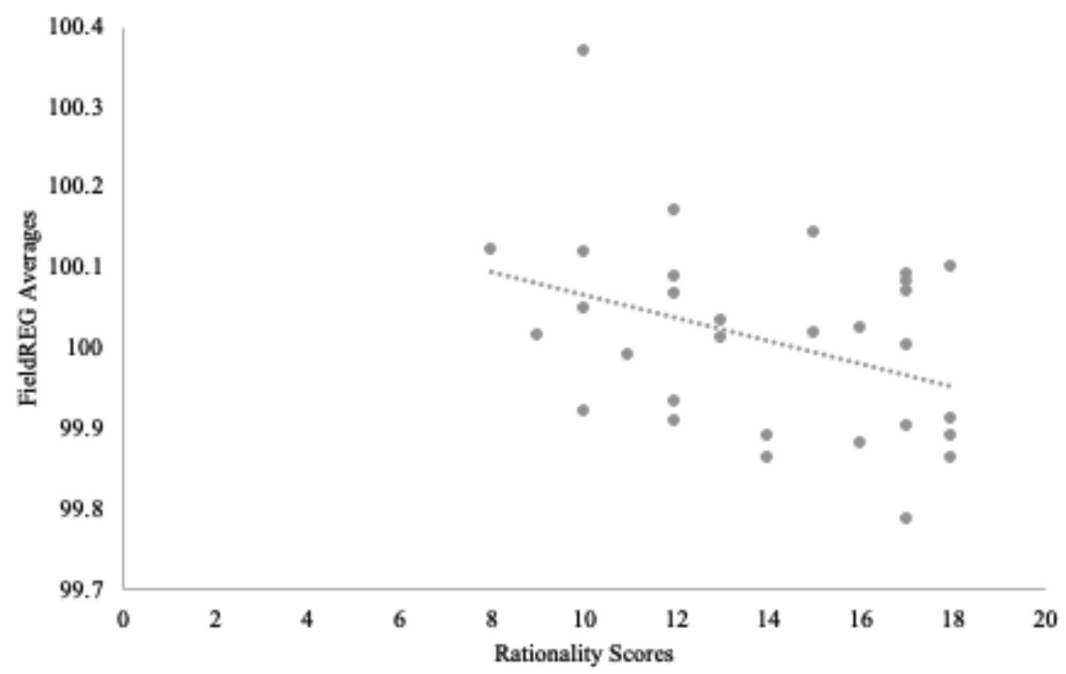

Figure 4. The correlation between rationality scores and FieldREG averages. As rationality scores increase, FieldREG averages appear to trend downward. 
that individuals who score high in rationality may be more likely to deviate the FieldREG in the negative direction. To appreciate the significance of this result, it is to be noted that only one of $21 \mathrm{PCl}$ scales was statistically significantly correlated with the FieldREG averages in Experiment 1 . That the same $\mathrm{PCl}$ scale is again the one that is statistically significant in Experiment 2 and in the same direction has a probability of only .024. This appears to be a signal in the noise.

A summary of the findings for the predictor variables in Experiment 2 can be found in Table 2 .

TABLE 2

Correlations, Significance, and Sample Size of Predictor Variables with the Difference Between High- and Low-Intention Runs

\begin{tabular}{lccc}
\hline \hline Predictor Variable & $\begin{array}{c}\text { Pearson } \\
\text { Correlation }\end{array}$ & $\begin{array}{c}\text { Significance } \\
\text { (two-tailed) }\end{array}$ & $\mathrm{N}$ \\
\hline $\begin{array}{l}\text { Love Subscale Score } \\
\text { Rationality Subscale }\end{array}$ & -.26 & .16 & 30 \\
Score & -.28 & .13 & 30 \\
\hline
\end{tabular}

\section{Discussion}

Results revealed a significant deviation of the REG in the direction of the participants' volition. Moreover, comparisons between cumulative deviations for high- and low-intention runs across both samples were found to be statistically significant. These findings offer support for the first hypothesis, indicating that the incorporation of meditation and visualization exercises prior to interaction with the REG may significantly influence an individual's ability to deviate the REG in the intended direction.

The results of Experiment 2 offer only partial support that feelings of love facilitate direct mental influence, and that rationality may create a deviation of the REG. However, mean love subscale scores gathered from the second administration of the $\mathrm{PCl}$ increased in Experiment 2. The manipulation in Experiment 2 was designed to initiate feelings of 
love, and participants in Experiment 2 were also found to be more likely to produce significant deviations in the intended direction. Perhaps, if there were more statistical power, then love subscale scores may have been a predictor for the mean difference between high- and lowintention runs overall.

It is important to note that an experimenter effect may have been present in Experiment 2 as two experimenters worked in collaboration to collect the data and the experiment required more direct interaction with the participants beyond the simple instruction given in Experiment 1. The meditation and visualization scripts were recited by one of the two experimenters, and visualization suggestions also were provided. Furthermore, the experimenters may have each played a role in directly altering the function of the REG. Upon investigation, there was no significant difference in the deviation scores between the two experimenters $F(2,57)=2.68, p=.08$ (two-tailed), although it is possible that both contributed equally to the elevated scores.

Another potential oversight was identified after half of the data had been collected as there was confusion regarding the correct method to fill out the $\mathrm{PCl}$. Specific instruction on how to fill out the inventory was not given for the first half of the participants in Experiment 2, and as a result statistical power may have been lost and some of the relationships that exist between the variables may have been missed.

\section{GENERAL DISCUSSION}

\section{Interpretations}

One explanation for the successful deviations in Experiment 2 is that the tension-releasing effects of the meditation component reduced anxiety levels sufficiently so as to not interfere with direct mental influence. As previously mentioned, individuals experiencing high levels of anxiety have been shown to produce psi-missing effects. In fact, creativity has been found to be linked with elevated psi-hitting scores; however, when creativity was accompanied by anxiety, the scores were negative (Carpenter, 2012).

Another plausible interpretation is that the visualization element, as well as the meditation component, may have encouraged participants to tap into their nonconscious intentions. Carpenter (2012) found that 
participants who presented a more active involvement in personal imagery, as well as an emotional openness to it, were more likely to produce extreme deviations. Participants who experienced imagery that contained high levels of intellectualization and cognitive analysis, on the other hand, were more likely to produce scores that did not deviate significantly in any direction. Rationality had a negative relationship with FieldREG averages in both experiments, and although there is no intention to deviate the REG in either direction, this may suggest that excessive cognitive analysis hinders psi-hitting effects.

As previously described, creativity has been associated with psihitting effects, and this may be due to the fact that the creative process requires a suspension of cognitive analysis for a sufficient amount of time so as to be able to access the nonconscious, our implicit sense, or for our purposes here, our nonconscious intentions (Carpenter, 2005). Meditation and visualization techniques promote creativity and an uninterrupted openness, and this may be how intentional stability at the psi level is maintained.

\section{Limitations}

Feedback. With regard to limitations, correlations with the love subscale on the $\mathrm{PCl}$ may be the result of a priming effect caused by the feedback given to participants regarding the results of their interactions with the REG. For example, if the mean increased or decreased in a manner consistent with the participant's intention, then this might result in the participant being in a more positive or "loving" mood. A solution to this would be to remove participant feedback.

Sampling Differences. Experiment 2 sampled fewer university students than Experiment 1 and included a larger proportion of participants who expressed interest in consciousness or belief in anomalous abilities. Thus, participants in Experiment 2 may have been more likely to produce successful deviations, due to individual characteristics as well. It is possible that these participants may have been more likely to produce a signification deviation in the intended direction because they were less anxious about, and more open to, psi phenomena.

Experimenter Effect and Psi-Missing. There are enigmatic aspects of studying direct mental influence, including psi-missing and the 
nonconscious reversal of conscious intentions, as well as experimenter effects. Not only may experimenters indirectly influence the collection of data, but the significant deviation in the intended direction that was found in Experiment 2 may have been in part or entirely due to the experimenters' psi-giftedness. Moreover, the inability to find a significant deviation in the intended direction for Experiment 1, may also be due to a possible experimenter effect.

\section{Conclusions}

As per Carpenter's First Sight Theory, psi phenomena are presumed to be nonconscious psychological processes that precede and shape all experience. Considering that these processes appear to function in a transient manner, they are difficult to investigate. A conservative evaluation of the evidence for psi phenomena is that results are statistically anomalous and further scientific inquiry is required; a more realistic evaluation is that anomalous phenomena are very likely occurring in the world around us, and to an extent that is beyond what can presently be explained by conventional science (Broderick \& Goertzel, 2014).

In fact, just because psi phenomena conflict with the current scientific understanding of reality, does not mean the results should be disregarded. There is an abundance of examples of newly discovered phenomena that have adjusted science's view of reality accordingly (Broderick \& Goertzel, 2014). For instance, Peter Higgs submitted a paper to a notable physics journal in 1964. The paper was rejected because it contained the premise of an invisible element pervading space and interacting with particles so as to supply their mass. What is of importance is that Higgs maintained his scientific position and his premises were eventually accepted (Broderick \& Goertzel, 2014). Despite our expectations for conventional explanations, conventional explanations, they may not always be the best ones for the phenomena occurring around us.

As a suggestion for future research, participants could be preselected if they were to exhibit some psi abilities, for example through the use of the Ball Selection Test (BST). In this test, a number from one through five is written on separate balls, and a participant is asked to guess the number on the ball that she will select from a bag 
containing an assortment of numbered balls. In one case, a sample of preschool children performing the BST yielded a high effect size (Broderick \& Goertzel, 2014). It was noted that the high effect size could have been due to the fact that the experimenter was exceptionally successful at the BST task, but this could also have been the result of children's creativity and imagination. Although the BST measures precognition and clairvoyance, it may nevertheless be useful for prescreening participants in experiments of direct mental influence.

Furthermore, current research concerning psi phenomena supports also preselecting participants on the basis of their acceptance of psi phenomena, previous transcendental experiences, the practice of meditation and visualization, and the possession of psychological characteristics such as extraversion, warmth, and love (Broderick \& Goertzel, 2014). The collective mental activity of individuals should also continue to be investigated with REG devices. Our understanding of consciousness and reality is in its infancy, and it is only through investigation and the accumulation of data that a scientific understanding of reality can be actualized.

\section{ACKNOWLEDGMENTS}

This research was supported by donations from Medical Technology (W. B.) Inc. and from internal research grants from King's University College. The authors thank their research assistants: Kasha Herba for contributing to the design of the second study and interacting with some of the participants, and Shannon Foskett for editing the manuscript of the paper. Experiment 1 was the first author's undergraduate honors thesis in psychology supervised by the third author; Experiment 2 was the second author's undergraduate independent study project supervised by the third author. The first author wrote up the paper for publication with assistance from the second and third authors.

\section{REFERENCES}

Barušs, I. (2007). Science as a spiritual practice. Imprint Academic.

Barušs, I. (2020). Alterations of consciousness: An empirical analysis for social scientists (2nd ed.). American Psychological Association. 
Barušs, I., \& Moore, R. J. (1992). Measurement of beliefs about consciousness and reality. Psychological Reports, 71(1), 59-64. https://doi.org/10.246/ pro.1992.71.1.59

Barušs, I., \& Moore, R. J. (1998). Beliefs about consciousness and reality questionnaire. Unpublished test manual.

Barušs, I., \& Mossbridge, J. (2017). Transcendent mind: Rethinking the science of consciousness. American Psychological Association.

Braud, W. (2003). Distant mental influence: Its contributions to science, healing, and human interactions. Hampton Roads Publishing.

Broderick, D., \& Goertzel, B. (2014). Evidence for psi: Thirteen empirical research reports. McFarland.

Carpenter, J. C. (1971). The differential effect and hidden target differences consisting of erotic and neutral stimuli. Journal of the American Society for Psychical Research, 65(2), 204-214.

Carpenter, J. C. (2004). First sight: Part one, a model of psi and the mind. The Journal of Parapsychology, 68(2), 217-254.

Carpenter, J. C. (2005). First sight: Part two, elaboration of a model of psi and the mind. The Journal of Parapsychology, 69(1), 63-112.

Carpenter, J. C. (2012). First sight: ESP and parapsychology in everyday life. Rowman \& Littlefield.

Dunne, B. J., \& Jahn R. G. (1992). Experiments in remote human/machine interaction. Journal of Scientific Exploration, 6(4), 311-332.

Dunne, B. J., Nelson, R. D., \& Jahn, R. G. (1988). Operator-related anomalies in a random mechanical cascade. Journal of Scientific Exploration, 2(2), 155-179.

Fox, K. C. R., Dixon, M. L., Nijeboer, S., Girn, M., Floman, J. L., Lifshitz, M., Ellamil, M., Sedlmeier, P., \& Christoff, K. (2016). Functional neuroanatomy of meditation: A review and meta-analysis of 78 functional neuroimaging investigations. Neuroscience and Behavioural Reviews, 65, 208-228. https:// doi.org/10.1016/j.neubiorev.2016.03.021

Jahn, R. G. (1996). Information, consciousness, and health. Alternative therapies, 2(3), 32-38.

Jahn, R. G., Dunne, B. J., \& Nelson, R. D. (1987). Engineering anomalies research. Journal of Scientific Exploration, 1(1), 21-50.

Jahn, R. G., Dunne, B. J., Nelson, R. D., Dobyns, Y. H., \& Bradish G. J. (1997). Correlations of random binary sequences with pre-stated operator intention: A review of a 12-year program. Journal of Scientific Exploration, $11(3), 345-367$.

Jahn, R. G., Dunne, B. J., \& Dobyns, Y. H. (2006). Exploring the possible effects of Johrei techniques on the behavior of random physical systems. Semantic Scholar Technical Report, 1-30. https://www.semanticscholar.org/paper/ Exploring-the-Possible-Effects-of-Johrei-Techniques-Jahn-Dunne/69743ao 


\section{1eggfi2a355049cabe $7 b f 2 d 78 c 8 a 3 a c f$}

Kuhn, T. S. (1970). The structure of scientific revolutions (2nd ed.). University of Chicago Press.

MacDonald, D. A. (2000a). Spirituality: Description, measurement, and relation to the five factor model of personality. Journal of Personality, 68(1), 153-197.

MacDonald, D. A. (200ob). The expressions of spirituality inventory: Test development, validation and scoring information. Unpublished test manual.

Margolin, I., Pierce, J., \& Wiley, A. (2011). Wellness through a creative lens: Meditation and visualization. Journal of Religion \& Spirituality in Social Work: Social Thought, 30(3), 234-252. https://doi.org/10.1080/15426432.2011.587385

Mertz, H. (2020). The selection effect: How consciousness shapes reality. Penn Wolcott Press.

Nelson, R. D., Bradish, G. J., Dobyns, Y. H., Dunne, B. J., \& Jahn, R. G. (1996). FieldREG anomalies in group situations. Journal of Scientific Exploration, 10 (1), 111-141.

Nelson, R. D., Jahn, R. G., Dunne, B. J., Dobyns, Y. H., \& Bradish G. J. (1998). FieldREG II: Consciousness field effects: Replications and explorations. Journal of Scientific Exploration, 12(3), 425-454.

Padfield, S. (1980). Mind-matter interaction in the psychokinetic experience. In B. S. Josephson \& V. S. Ramachandran (Eds.), Consciousness and the physical world: Edited proceedings of an interdisciplinary symposium on consciousness held at the University of Cambridge in January 1978 (pp. 165-175). Pergamon Press.

Pekala, R. J. (1991a). Quantifying consciousness: An empirical approach. Plenum Press.

Pekala, R. J. (1991b). The phenomenology of consciousness inventory. Mid-Atlantic Educational Institute.

Psyleron. (2009). REG-1 user manual. Psyleron Technologies and Research. Psyleron.com Radin, D., Michel, L., Galdamez, K., Wendland, P., Rickenbach, R., \& Delorme, A. (2012). Consciousness and the double-slit interference pattern: Six experiments. Physics Essays, 25(2), 157-171.

Schmidt, H. (1971). Mental influence on random events. New Scientist and Science Journal, 50(757), 757-758.

Varvoglis, M. P., \& McCarthy, D. (1986). Conscious-purposive focus and PK: RNG activity in relation to awareness, task-orientation, and feedback. Journal of the American Society for Psychical Research, 80(1), 1-29.

Walach, H., Horan, M. Hinterberger, T., \& von Lucadou, W. (2020). Evidence for anomalistic correlations between human behavior and a random event generator: Result of an independent replication of a micro-pk experiment. Psychology of Consciousness: Theory, Research, and Practice, 7(2), 173-188. 


\section{APPENDIX A RELAXATION MEDITATION SCRIPT}

I will now guide you through some imagery exercises for the purpose of allowing you to relax more deeply. Begin by settling your body into a comfortable sitting position and allowing your eyes to gently close. ... Let your awareness shift to your breathing. ... Allow yourself to let go of any thoughts or concerns that may have accumulated during the day. ... Simply direct your focus on what you are experiencing here and now. ... As you continue to breathe, observe your body and scan for any areas that may feel tense. You may notice some tension in your neck or shoulders.... Your back. ... Feet. ... Or perhaps, your jaw feels tight. ... Wherever the tension lies, direct attention to the source and focus on releasing any tension that may feel stuck in this particular area. ... With each outbreath, consciously say to yourself, "release." ... Imagine the tension being washed away. . . . Continue to breathe. ... and feel the relief that follows as the tension is released from this area.... When you feel ready to move on, scan your body once again and select another area that may feel tense. ... Direct your attention towards the source of this tension. . . Again as you exhale, consciously say to yourself "release" ... and imagine the area being cleared of all tension. ... Over the next few breaths, focus on releasing tension from this part of your body and allow this area to feel relaxed and soothed.

\section{APPENDIX B VISUALIZATION EXERCISE SCRIPT}

We will now focus on tapping into our imagination through the process of visualization. If the practice of visualization is new to you or you find that the images are unclear in your imagination, feel free to follow along and imagine the sequence of events in whatever way is most comfortable to you. We will begin very simply by walking. Imagine yourself walking through a lush forest. It's the break of dawn in early summer. ... As the sun rises it paints the sky with the most vibrant hues. Splashes of orange, purple, yellow, and pink are reflected in the big, fluffy clouds above as beams of white light radiate through each opening. The air feels brisk, but you feel perfectly comfortable. The soft cozy sweater you are wearing is keeping you nice and warm. As you breathe deeply, filling your lungs with the cool fresh air, you notice how rejuvenating it feels to do so, almost as though the forest were cleansing you in some way. As you continue walking, you notice the sun periodically peeking through the treetops to shine down upon you. You stop for a moment to feel the warmth of the sun's rays on your face. Let the feeling sink in. ... As you pause there, basking in the sunlight, you happen to notice the soft sound of birds chirping 
in the trees and the rustle of leaves in a gentle breeze, ... but there is mostly silence. ... You notice how peaceful the absence of sound can be. There is a new level of clarity. As you continue on your journey, you glance down at your feet and notice that the path beneath you is made of soft, warm sand. You wiggle your toes. That feels good. As you look up you notice a clearing up ahead ... and begin walking towards the opening in the forest. As you walk, you look around and admire the rich colours of the plants and flowers around you. The forest feels alive. You bend down to examine a flower ... noticing its colours, caressing its leaves, drinking in the scent emanating from its blossoms. The fresh, sweet smell stays with you as you continue along the path. As you near the clearing, you notice that there is someone waiting to greet you. As you get closer, you recognize that this is a person you know who loves you very deeply. This can be someone real or imaginary. The person is smiling at you and waving you over. You pick up the pace ... and feel joy welling up inside of you as your legs move faster and faster ... moving you closer and closer. You finally reach each other, and they wrap you in their arms. They tell you how proud they are of you and what you are creating in this world. You take a moment to look at each other, before walking down to a nearby pond. As you take a seat on the grass, you see frogs leaping from their lily pads and splashing into the water. You take a deep breath and as you exhale, once again notice the sun shining down on you and warming your cheeks. You think to yourself, what a peaceful day. ... When you are ready to do so, return to the room and open your eyes.

\section{APPENDIX C VISUALIZATION SUGGESTIONS}

During this stage of the experiment your goal will be to intend the center line to move up (for a high-intention run) or down (for a low-intention run). There will be a total of 20 runs; 10 of which must be high, which will appear as red lines, while the other 10 must be low, which will appear as blue lines. At the beginning of each run, you will have the option of selecting which direction you wish to set your intention. If you would like to intend the line to move up on the screen, it may be beneficial to imagine that the line has a weightless quality to it ... or that it is taking off towards the top of the screen. Similarly, if you would like to intend the line to move down, it might help to imagine that there is a heavy quality to the line ... or that it is sinking down to the bottom of the screen. While initially setting your intention is important, it is best not to become too absorbed in the small, inevitable fluctuations that the line will make during the course of each run. Simply focus on the big picture and the intended direction you would like the line to move. When you are ready, we can begin. 


\section{APPENDIX D \\ DEMOGRAPHICS AND ATTITUDES FORM}

Name:

Age:

Gender: $\square$ Female $\square$ Male

Highest level of education:

High School Graduate $\square$ College Graduate $\square$ Post-graduate Degree $\square$ None of these

\section{Religious Affiliation:}

Buddhist $\square$ Judaic $\square$ Christian $\square$ Muslim $\square$ Own Beliefs $\square$ None Other (Please specify):

Frequency of religious practice:

Daily $\square$ Weekly $\square$ Monthly $\square$ Hardly Ever $\square$ Never

\section{Attitude toward REG:}

Please rate your agreement with the following statement:

I think that I can alter the functioning of the REG.

$\square$ definitely not $\square$ likely not $\square$ don't know $\square$ likely yes $\square$ definitely yes

\section{APPENDIX E}

\section{QUESTIONS ABOUT THE EXPERIENCE WITH THE RANDOM EVENT GENERATOR}

1. Please describe your experience with the random event generator (REG).

\section{Attitude toward REG:}

Please rate your agreement with the following statements:

2. I think that I was able to alter the functioning of the REG.

$\square_{\text {definitely not }} \square$ likely not $\square$ don't know $\square$ likely yes $\square$ definitely yes

3. I felt that I was one with the REG.

definitely not $\square$ likely not $\square$ don't know $\square$ likely yes $\square$ definitely yes 